REVIEW ARTICLE

\title{
Application of combinatorial optimization strategies in synthetic biology
}

\author{
Gita Naseri (iD ${ }^{1 凶} \&$ Mattheos A. G. Koffas (1D ${ }^{2,3,4 凶}$
}

In the first wave of synthetic biology, genetic elements, combined into simple circuits, are used to control individual cellular functions. In the second wave of synthetic biology, the simple circuits, combined into complex circuits, form systems-level functions. However, efforts to construct complex circuits are often impeded by our limited knowledge of the optimal combination of individual circuits. For example, a fundamental question in most metabolic engineering projects is the optimal level of enzymes for maximizing the output. To address this point, combinatorial optimization approaches have been established, allowing automatic optimization without prior knowledge of the best combination of expression levels of individual genes. This review focuses on current combinatorial optimization methods and emerging technologies facilitating their applications.

$\mathrm{n}$ the past decade, synthetic biologists have built an impressive collection of elements and tools (genetic sequences performing defined functions such as promoters) and combined

them to achieve circuits with more advanced functions e.g. transcriptional regulation. These circuits are now being combined to design regulatory circuits with complex performance, such as logical gates ${ }^{1}$, RNA riboswitches ${ }^{2,3}$, oscillators ${ }^{4}$, and recorders ${ }^{5}$.

However, despite great developments of cutting-edge synthetic biology tools, engineering microorganisms for industrial scale production is still a challenging effort, even for well-known metabolic pathways ${ }^{6}$. Often, multiple genes must be introduced into the host and expressed at appropriate levels to achieve the best possible output. Due to the enormous complexity of living cells, it is typically unknown at which level heterologous genes must be expressed, and to which level the expression of host-endogenous genes must be altered (if not deleted), to accomplish the goal $^{7}$. Therefore, synthetic biologists aim to develop computational tools that can allow prediction of the performance of an assembly or an entire recombinant microorganism ${ }^{8,9}$. However, results from the computational analysis typically require validation through further wet-lab testing. This difficulty principally stems from the nonlinearity of biological systems ${ }^{10}$ and lowthroughput characterization methods ${ }^{11}$. Furthermore, it is not always clear how to control noise ${ }^{12}$ and how to transfer the functionality of engineered elements between organisms ${ }^{7}$. Moreover, tweaking multiple factors can typically be critical to obtain an optimal output in a biological system ${ }^{13}$. Those may include overall structural state of chromatin and its domains ${ }^{14}$, the strength of transcriptional regulators controlling gene expression ${ }^{8,15}$, transcriptional terminators ${ }^{16-18}$, ribosome binding sites (RBS), biochemical properties of the protein(s) encoded by the recombinant genes ${ }^{19-21}$, the availability of cofactors for the correct functionality of

\footnotetext{
${ }^{1}$ Institut für Chemie, Humboldt Universität zu Berlin, 12489 Berlin, Germany. ${ }^{2}$ Center for Biotechnology, Rensselaer Polytechnic Institute, Troy, NY, USA. ${ }^{3}$ Department of Chemical and Biological Engineering, Rensselaer Polytechnic Institute, Troy, NY, USA. ${ }^{4}$ Department of Biological Sciences, Rensselaer

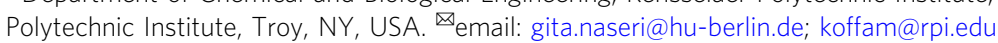


enyzmes $^{22,23}$, the genetic background of the host ${ }^{24-26}$, and the expression system itself (plasmid-based vs. chromosomal integration $)^{27}$.

To overcome these issues, two types of optimization strategies are available. The first one is "sequential optimization", a classic method to optimize pathway performance ${ }^{28}$. The sequential flux maximization methodologies frequently utilize deletion of genes encoding competing pathways ${ }^{29}$. However, deletion of a gene can have broad physiological consequences that decrease cellular growth and productivity. For example, different levels of ArgR downregulation, achieved by CRISPR interference (CRISPRi), resulted in two times higher growth rates of Escherichia coli compared to deletion of $\operatorname{ArgR}^{30}$. However, increasing the production rate of a heterologous product in a recombinant microorganism is complicated and narrowing the research to debottlenecking strategies is too much of a simplification. For example, extensive work has been conducted to investigate the metabolism of the budding yeast Saccharomyces cerevisiae; nonetheless, there is still little progress in industrial scale production of high-value chemicals in this organism ${ }^{31}$. In one example, 244,000 synthetic DNA sequences were recently designed to uncover design principles of optimized translation in the well-known prokaryotic host E. coli ${ }^{32}$. Although impressive, this work provided little information about possible mechanisms underlying the improved translation capacity.

Using sequential optimization, only one part, or a small number of parts, is tested at a time, making the approach timeconsuming and expensive $e^{12,33}$ and successful engineering of pathways is usually achieved only by trial-and-error ${ }^{7}$. Another approach to circumvent these barriers is to establish pathway "optimization" methods that do not require prior knowledge of the optimal expression levels of each individual gene involved in a multi-enzyme pathway. Several such methods have recently been developed, such as the functional optimization of gene clusters ${ }^{34}$, perturbation of the global transcription machinery ${ }^{35}$, genomicscale mapping of fitness modifying genes ${ }^{36}$, multiplex automated genome engineering 37,38 , and "combinatorial optimization". Jeschek et al. defined combinatorial optimization as "multivariate optimization" (in the context of metabolic engineering) 7 . The combinatorial optimization allows the rapid generation of a large number of diverse genetic constructs in short time ${ }^{7}$. Later on, to achieve high-level production of metabolites, microbial strains in a library that produce the highest level of a metabolite of interest need to be identified (Fig. 1) 39,40 .

Here, we present recent advances in synthetic biology tools that enable the development of complex libraries, and we summarize combinatorial optimization strategies that have been developed recently. Next, we discuss the application of barcoding tools to facilitate tracking diversity to streamline combinatorial optimization techniques. Furthermore, we discuss the application of "biosensors" for high-throughput screening used within the frame of combinatorial optimization. We highlight development in computational and machine learning methods to help generate optimal constructs through minimizing or maximizing target functions out of a defined subject. Finally, we outline applications of combinatorial pathway optimization methods beyond metabolic engineering.

\section{From combinatorial optimization to efficient production}

Synthetic biology tools ${ }^{41}$ and design principles (Fig. 2, black arrow) are being used to accelerate development of combinatorial optimization methods. The barcoding tools ${ }^{42}$ can next be used to study the versatility of combinatorial optimization techniques at DNA level (Fig. 2, gray arrow). However, the identification of microbial strains in a library that produce the highest level of a
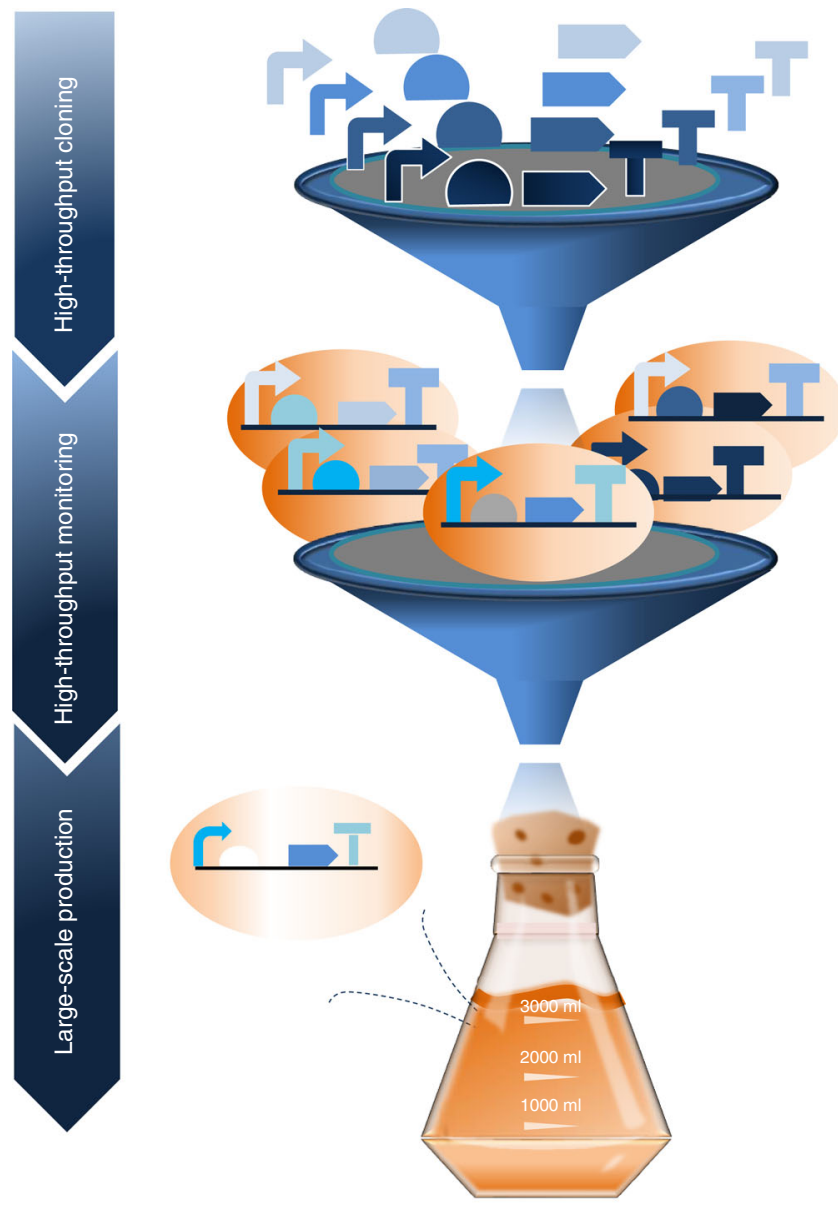

Fig. 1 Schematic workflow for microbial factory optimization. Libraries of pathway elements such as promoters (bent arrow), RBSs (chord), coding sequences (arrow), terminators ("T") are assembled to generate a combinatorial library, in which the microbial members produce different levels of the target metabolite. High-throughput techniques screen the library for the optimized pathway variant. Consequently, the best producer is used for large-scale production.

metabolite of interest often remains a laborious task, mainly due to time-consuming metabolite screening techniques ${ }^{39,40}$. To address this issue, the genetically encoded whole cell "biosensors" and the laser-based flow cytometry technologies are combined to transduce the production of chemicals into easily detectable fluorescence signal (Fig. 2, blue arrow) ${ }^{5}$.

Generating combinatorial library. Combinatorial cloning methods aim to generate multigene constructs from libraries of standardized basic genetic elements such as regulators, gene coding sequences, and terminators using a series of one-pot assembly reactions ${ }^{8,39}$. In Fig. 3, we illustrated a tailor-made pipeline for a complex combinatorial library generation. The workflow starts with in vitro construction and in vivo amplifying of combinatorially assembled DNA fragments to generate gene modules. Terminal homology between adjacent assembly fragments and the plasmids allows generating diverse construct in single cloning reaction. In each module, the gene expression is controlled by library of regulators ${ }^{39}$. To decrease the turnaround time in bioengineering projects, CRISPR/Cas-based editing strategies are implemented for multi-locus integration of multiple 

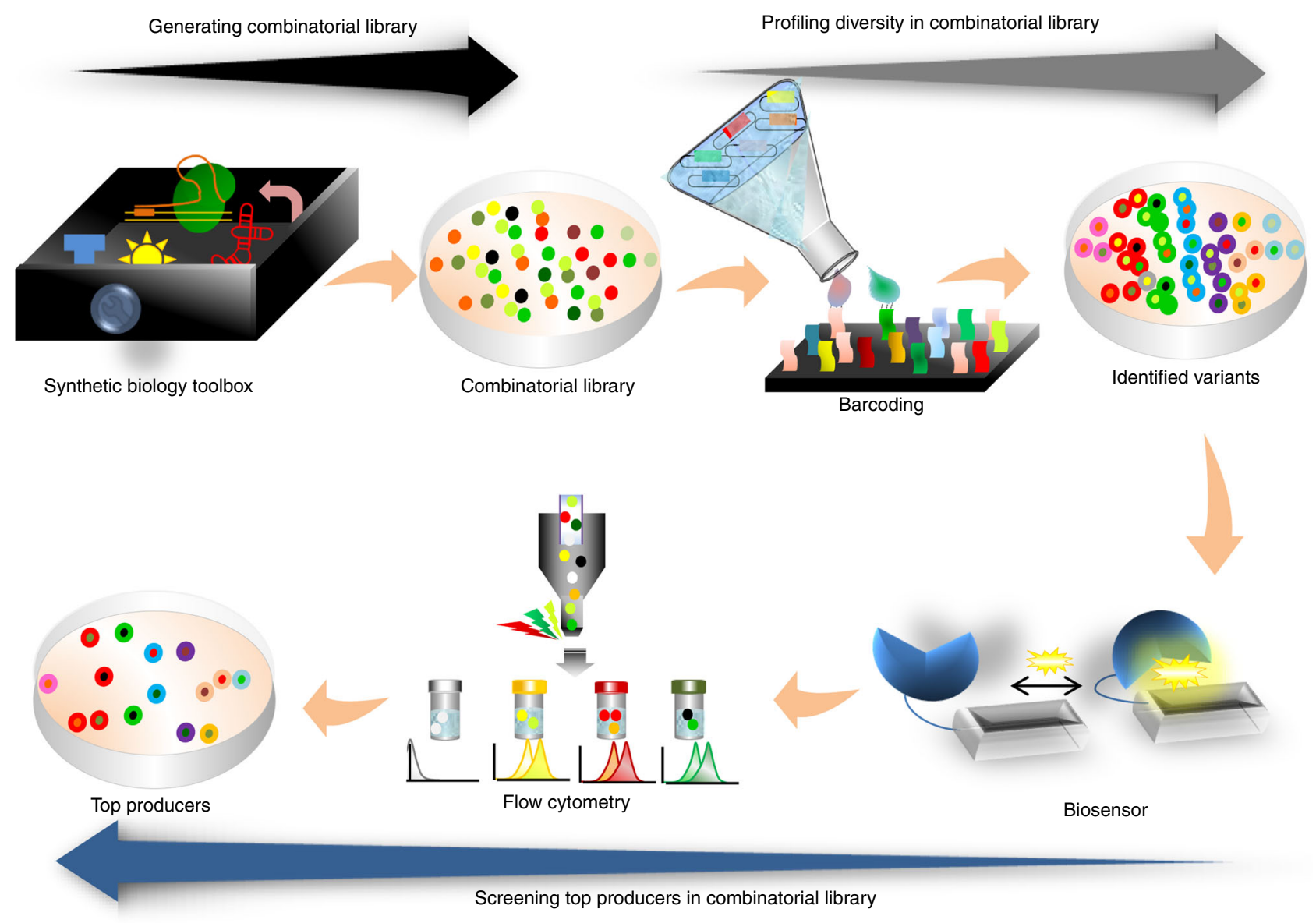

Fig. 2 Applying synthetic biology tools toward optimized production of chemicals. Synthetic biology speeds up combinatorial optimization. DNA modification tools in the synthetic biology toolbox provide combinatorial optimization methods with various tools e.g. regulators and genome editing tools (black arrow). Barcoding allows tracking of combinatorial library members through screening steps (gray arrow). Biosensors paired with high-throughput monitoring techniques, such as flow cytometry, improve selection of library members to isolate (blue arrow).

groups of modules into loci, whereby each group is integrated into a single locus of different microbial cells ${ }^{39}$. Sequential rounds of cloning enable the construction of entire pathway in a plasmid. The established plasmid can be either transformed into the host (e.g. VEGAS method ${ }^{43}$ ) or be used for single- or multi-locus integration into the microbial host genomes to generate combinatorial library (e.g. COMPASS ${ }^{39}$ ). Therefore, combinatorial optimization projects require tools and methods to assemble parts in genetic circuits, to change DNA sequences, and to integrate DNA pieces into the genome of an organism ${ }^{31}$. Here, we discuss two important synthetic biology tools: "advanced orthogonal regulators" and "advanced genome-editing tools", as well as the recently established combinatorial optimization strategies.

Advanced orthogonal regulators. Constitutive promoters are typically used to express heterologous genes in microbial cell factories, but this is often metabolically burdensome as the formation of the product competes with cell growth and proliferation $^{8}$. Hence, auto-inducible (or self-induction) protein expression systems were established to pair growth and induction of recombinant proteins at desired time ${ }^{44}$. The system utilizes a cell density-based control module that allows tight regulation of the transcription of the recombinant gene; at low cell density, its expression is negligible while at high cell density expression is high. Such cell density-based control systems usually employ the quorum sensing (QS) mechanisms from Vibrio fischeri implemented in E. coli. However, these systems require the use of an extra plasmid for the production of proteins under control of the regulatory elements, which might be undesirable, particularly when multiple genes must be expressed. Another way of controlling the expression of regulators takes advantage of phagederived anti-CRISPR proteins that allow fine-tuning the activity of dCas9-derived regulators at desired time points. The antiCRISPR molecule inhibits the binding of Cas9 protein to DNA. This allows cells become resistant to further gene editing ${ }^{45}$. In another work, $\beta$-farnesene, pantothenate (a metabolic precursor of coenzyme A) was developed as a metabolic switch that effectively postpones metabolic burden until an optimal time for achieving maximal yield is reached ${ }^{46}$. Cultivation in media lacking pantothenate removes the growth advantage of lowproducing mutants, resulting in improved production upon a scale-up to lab-scale bioreactors.

Small RNAs can also be used to control the expression of genes by RNA-DNA or RNA-RNA interactions ${ }^{47}$. More specifically, small RNAs can affect the chromatin structure allowing regulation of gene expression at the transcriptional level, or can affect mRNA stability post-transcriptionally or during translation. As a more sophisticated solution, orthogonal (and inducible) ATFs have been developed recently to control the timing of gene expression in various microorganisms ${ }^{48}$. To generate ATFs, the DNA binding domains (DBDs) of zinc finger proteins (ZFPs) ${ }^{49}$, transcription activator-like effectors (TALEs) ${ }^{15}$, and CRISPR/ dCas $9^{9,15}$ scaffolds are used. Other DBDs, including LexA ${ }^{50}$, $\mathrm{SrpR}^{50}, \mathrm{PhlF}^{50}, \mathrm{TarA}^{50}, \mathrm{Bm} 3 \mathrm{R} 1^{50}$, TetR ${ }^{50}$, auxin based degron ${ }^{51}$, FadR $_{\mathrm{Sa}}$, CarH ${ }^{52}$, and plant $\mathrm{TFs}^{8}$ may be promising alternatives. 

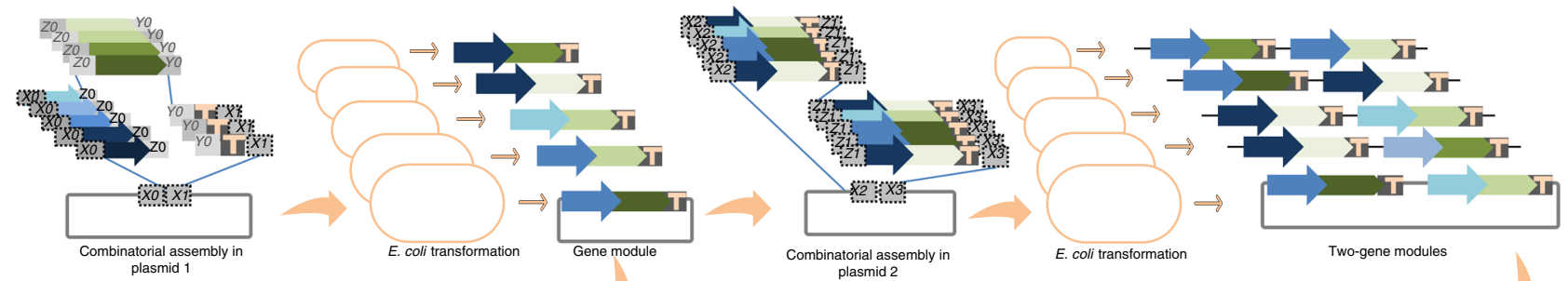

plasmid 1
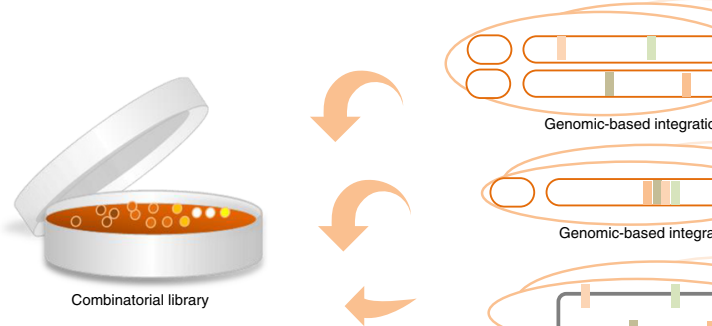

Genomic-based integration

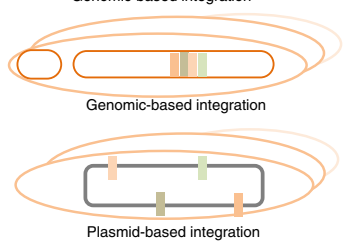

Gene
Regulator

T. Terminator
: $\quad 5^{t}$ (left)- sequence of plasmid 1

$3^{4}$ (right)- sequence of plasmid 1 $x^{2}: \quad 5^{4}$ (left)- sequence of plasmid 2

$3^{4}$ (right)- sequence of plasmid 2
E. coli transformation

Two-gene modules
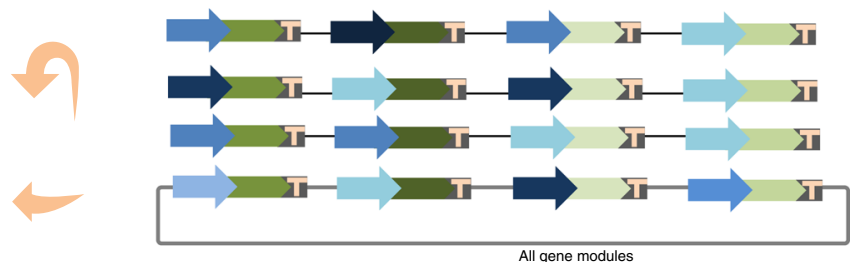

ZO Identical sequence between regulator and gene fragments

$\begin{array}{ll}\text { YO Identical sequence between gene and terminator frag } \\ Z 1 & \text { Identical sequence between gene modules } 1 \text { and } 2\end{array}$

Fig. 3 Schematic workflow to generate complex combinatorial library. Construction of a combinatorial library relies on iterative engineering cycles of onepot assembly reactions, and amplification of assembled products in microbial cells. At the level of the assembly reaction, the reaction cocktail contains libraries of genetic elements such as promoters (blue arrow), genes (green arrow), and terminators (orange "T"). Combinatorial assembly allows assembly of all standard elements (e.g. promoters, genes, and terminators) in different combination in a single cloning step. To do this, homology sequences (for homology-based cloning method) or sequences that consist of a restriction enzyme cleavage site (for classical digestion/ligation method) at the ends of the fragments to assemble are required: $X 0$ and $X 1$ are segments upstream (left) and downstream (right) of the cloning in plasmid 1, respectively; segment $Z O$ represents the $3^{\prime}$ region of the promoter and overlaps with the sequence upstream (left) of the gene; segment YO represents the $3^{\prime}$ region of the gene and overlaps with the sequence upstream (left) of the terminator. Thereafter, the multiple groups of gene modules of may be integrated into multilocus of the host genome. A first combinatorial reaction cocktail is used for assembly of gene module, while a second reaction is used for generation of two-gene module from individual gene module in plasmid 2. $X 2$ and $X 3$ are segments upstream (left) and downstream (right) of the cloning in plasmid 2, respectively; and segment $Z 1$ represents the $3^{\prime}$ region of the first gene module and overlaps with the sequence upstream (left) of the second gene module. After establishing a plasmid library containing the entire pathway gene modules, the plasmid library can be directly transformed into the host or can be integrated into the genome of the host to generate stable combinatorial library variants.

However, unlike DBDs from TALE and CRISPR/dCas9 proteins, these DBDs cannot be easily modified to target any desired genetic sequence because they need to be paired with their own specific binding site. Nevertheless, ATFs have so far rarely been used in metabolic engineering projects due to their often large molecular size or low transcriptional activity. Surprisingly, plantderived ATFs have recently been developed as strong regulators for $S$. cerevisiae (10-fold stronger than the yeast constitutive and strong TDH3 promoter $)^{8}$. Expression of ATFs can be controlled by either exogenous chemical inducers (e.g., IPTG, arabinose) ${ }^{8}$ or by light of specific wave lengths ${ }^{53}$. However, hypersensitivity, toxic and pleiotropic effects limit the utilization of chemical inducers. An important task of future research is the identification of cost-effective inducers (chemical or others) that allow to control and modulate protein levels in response to a defined input signal in a fast-acting, tunable and robust manner. In this regard, light-based (i.e., optogenetic) systems have been developed that allow the expression of a gene of interest to an anticipated level by exposing the metabolite producing cells to short light pulses. Previous reviews have described diverse optogenetic control systems $^{54}$. In Table 2, we list reports about different lightinducible systems, DBDs, transcription activation/repression domains (A/RDs), and the light types utilized to regulate their expression in various hosts, published since 2012. To the best of our knowledge, light-inducible systems have not yet been employed in combinatorial optimization methods. Rapamycin and its synthesized analogues are commonly used chemical inducers of dimerization (CIDs) employed by chemical biologists to place biological processes under conditional control. These CIDs bind to FK506 binding protein (FKBP) with a remarkably tight binding affinity. This FKBP-rapamycin complex then binds to the FKBP binding domain of mTOR (FRB) ${ }^{55}$. Such complexes have been largely employed as heterodimerization tools for small molecule switches. Unlike photoswitchable protein dimerizers (Table 1), FKBP and FRB are significantly smaller molecule photoswitches and do not need pulsed (or even constant) irradiation $^{55}$, allowing minimum potential for phototoxicity. Considering the recent progress in the area of new light-sensing proteins $^{56}$ and light-inducible circuit building 55,57 , lightcontrolled systems are more likely to be able to orthogonally regulate the expression of genes compared to chemical-dependent systems.

Advanced genome-editing tools. Chromosomal pathway integration projects are classically divided into multiple steps because the rate of native double-strand breaks (DSB) followed by homology-directed repair (HDR) is not high enough to support simultaneous generation of large numbers of integrations, even in a suitable host like $S$. cerevisiae. To greatly increase the recovery of HDR-based genetic engineering events, selection markers are often included in DNA integration cassettes. Recycling the marker genes, after their initial integration into the genome, allows cells to become competent for the next round of pathway engineering, as markers can be "re-used". The Cre-LoxP system ${ }^{51,57,58}$ and CRISPR/Cas9-mediated genome modification can be utilized to remove or mutate the selection marker coding sequences ${ }^{59}$. 
Table 1 Various light-inducible systems developed since 2012.

\begin{tabular}{|c|c|c|c|c|c|}
\hline Light & DBD & A/RD & Photoreceptor and partner & Host & Reference \\
\hline Blue & ZFP & VP16 AD & LOV and $\mathrm{Gl}$ & Human cell & Polstein et al. ${ }^{109}$ \\
\hline Red & TALE & VP64 AD & Cry2 and CIB1 & Mammalian cell & Konermann et al. ${ }^{110}$ \\
\hline Red & Gal4 & VP16 AD & PhyB and PIF3 & Mammalian cell & Müller et al. ${ }^{111}$ \\
\hline UV-B & & & UVR8 and COP1 & & \\
\hline Blue & & & LOVpep and PDZ & & \\
\hline Red & TetR & VP16 AD & PhyB and PIF3 & Plant cell & Müller et al. ${ }^{112}$ \\
\hline Blue & CRISPR/Cas 9 & VP64 AD & Cry2 and CIB1 & Mammalian cell & Polstein et al. ${ }^{113}$ \\
\hline Blue & CRISPR/Cas9 & P65 AD & Cry2 and CIB1 & Mammalian cell & Nihongaki et al. ${ }^{114}$ \\
\hline Blue & LexA & VP16 AD & Cry2 and CIB1 & S. cerevisiae & Taslimi et al. ${ }^{115}$ \\
\hline Red & TALE & VP64 AD & PhyB and PIF3 & S. cerevisiae & Hochrein et al. ${ }^{53}$ \\
\hline Red & TALE & VP64 AD & PhyB and PIF3 & S. cerevisiae & Hochrein et al. ${ }^{57}$ \\
\hline Blue & $\mathrm{N}$ terminal-T7 RNAPs & C terminal-T7 RNAPs AD & nMag and pMag & E. coli & Baumschlager et al. ${ }^{116}$ \\
\hline Blue & LexA & Gal4 AD & ClpX and ClpP & S. cerevisiae & Xu et al. ${ }^{117}$ \\
\hline Blue & Gal4 & Gal4 AD & WC-1 and VVD & S. cerevisiae & Salinas et al. ${ }^{118}$ \\
\hline Blue & TetR & P65 AD & Cry2 and CIB1 & Plant cell & Yamada et al. ${ }^{119}$ \\
\hline Green & AdoB12 & VP16 AD & $\mathrm{CarH}$ and $\mathrm{CarO}$ & Plant cell Mammalian cell & Chatelle et al. 52 \\
\hline Blue & ZFP & VP16 AD & CRY2 and CIB1 & S. cerevisiae & An-adirekkun et al. ${ }^{120}$ \\
\hline Blue & NLS & dCas9 RD & LOV and $\alpha$ helix & S. cerevisiae & Geller et al. ${ }^{121}$ \\
\hline
\end{tabular}

However, iterative rounds of engineering long pathways are time consuming.

A alternative is the CRISPR-Cas9 system (RNA-guided genome editing tool) which induce DSB and therefore allow higher efficiency in genome editing ${ }^{60-63}$. The combinatorial optimization method COMPASS allows the generation of a library of stable $S$. cerevisiae variants with thousands to millions of different members through only four cloning reactions followed by a one-step decoupled CRISPR-Cas9-mediated integration of the variants into the genome $\mathrm{e}^{39}$. In a recent study, a method for the manipulation of the genome of mammalian cells was established through combining the CRISPR-Cas9 and the Cre-Lox systems ${ }^{64}$. In another effort, two optogenetic recombinases were developed for E. coli ${ }^{65}$. This approach uses split Cre and Flp (originally native to $S$. cerevisiae) recombinases coupled with photodimers, where blue light brings the split protein together to form a functional recombinase. However, application of DSB-mediated genome editing is limited by the cellular cytotoxicity of HDR, unwanted DNA insertions and deletions, or unwanted additional DSB. To overcome these limitations, Barbieri et al. developed eMAGE (eukaryotic Multiplex Automated Genome Engineering) to precisely modify multiple sites of a genome without an involvement of DSBs. The method utilizes synthetic single-strand DNA (ssDNA) oligonucleotides targeting the lagging strand of the desired gene(s) in the replicating chromosome. Lambda $(\lambda)$ phage Red Beta ssDNA annealing protein facilitates annealing of the ssDNA oligonucleotides to the lagging strand of the targeted DNA during DNA replication at the replication fork in S. cerevisiae ${ }^{66}$. Genome editing area of research can (i) shorten the gap between genome engineering and combinatorial library construction and (ii) the generation of diverse cell variants.

Combinatorial optimization strategies. A multi-gene pathway can be combinatorially optimized at the DNA level by altering gene copy number, at the mRNA level by controlling transcriptional output and transcript stability, at the protein lLianevel by modifying translational capacity, protein post-translational modification, protein stability, and protein co-localization, at the metabolite level by dynamic metabolic flux control and at the chassis level by engineering microbial consortia. Lian et al. developed a combinatorial strategy based on an orthogonal tri-functional CRISPR system that combines transcriptional activation, transcriptional interference, and gene deletion (CRISPR-AID) for metabolic engineering purposes in S. cerevisiae ${ }^{67}$.

The majority of the research in the combinatorial optimization area has focused on transcriptional control mechanisms as an important control point for pathway gene expression. Currently available combinatorial approaches typically employ constitutive promoters, and therefore rely on growth-coupled biosynthesis. Versatile genetic assembly system (VEGAS) exploits the native capacity of $S$. cerevisiae to perform homologous recombination and efficiently join sequences with terminal homology. Yeast Golden Gate (yGG) is used to build transcription units (constitutive promoter upstream of a pathway gene) for VEGAS assembly. In the yGG reaction, each transcription unit is assigned a pair of VEGAS adapters that assemble up- and downstream of each transcription unit; VEGAS adaptor sequences subsequently provide terminal homology for overlap-directed assembly by homologous recombination "in yeast". In contrast, inducible regulators allow conditional control of heterologous gene expression. Only a limited number of methods employing inducible and modular transcription factor-based controllers have been established so far, including e.g. ePathOptimize ${ }^{68}$ and COMPASS $^{39}$. Combinatorial approaches typically rely on plasmid-derived systems to take advantage of the simplicity of manipulation at the plasmid level. However, genomic integration of a metabolic pathway leads to higher and more stable production of a target chemical than expression of genes from plasmids 8,53 , without the need for expensive selectable markers. In general, the chosen production host "determines" the regulatory elements (promoters, terminators, RBS), codon preferences, the way enzymes (or proteins in general) are modified by posttranslational modification (e.g., phosphorylation), and the biochemical mechanisms by which enzymes or metabolites are secreted out of the cell and into the growth medium (an important aspect of synthetic biology applications). Established combinatorial pathway optimization methods for metabolic engineering are summarized in Table 2. COMPASS, the most recently established approach for combinatorial optimization in the $S$. cerevisiae, employs inducible plantderived artificial transcription factors (ATFs) and utilizes three technical settings, (i) multi-locus integration of multiple genes into the yeast genome, when speed of strain development is prioritized, (ii) plasmid-based systems, when easy manipulation is favorable, and (iii) single genomic locus integration of multigene constructs, 


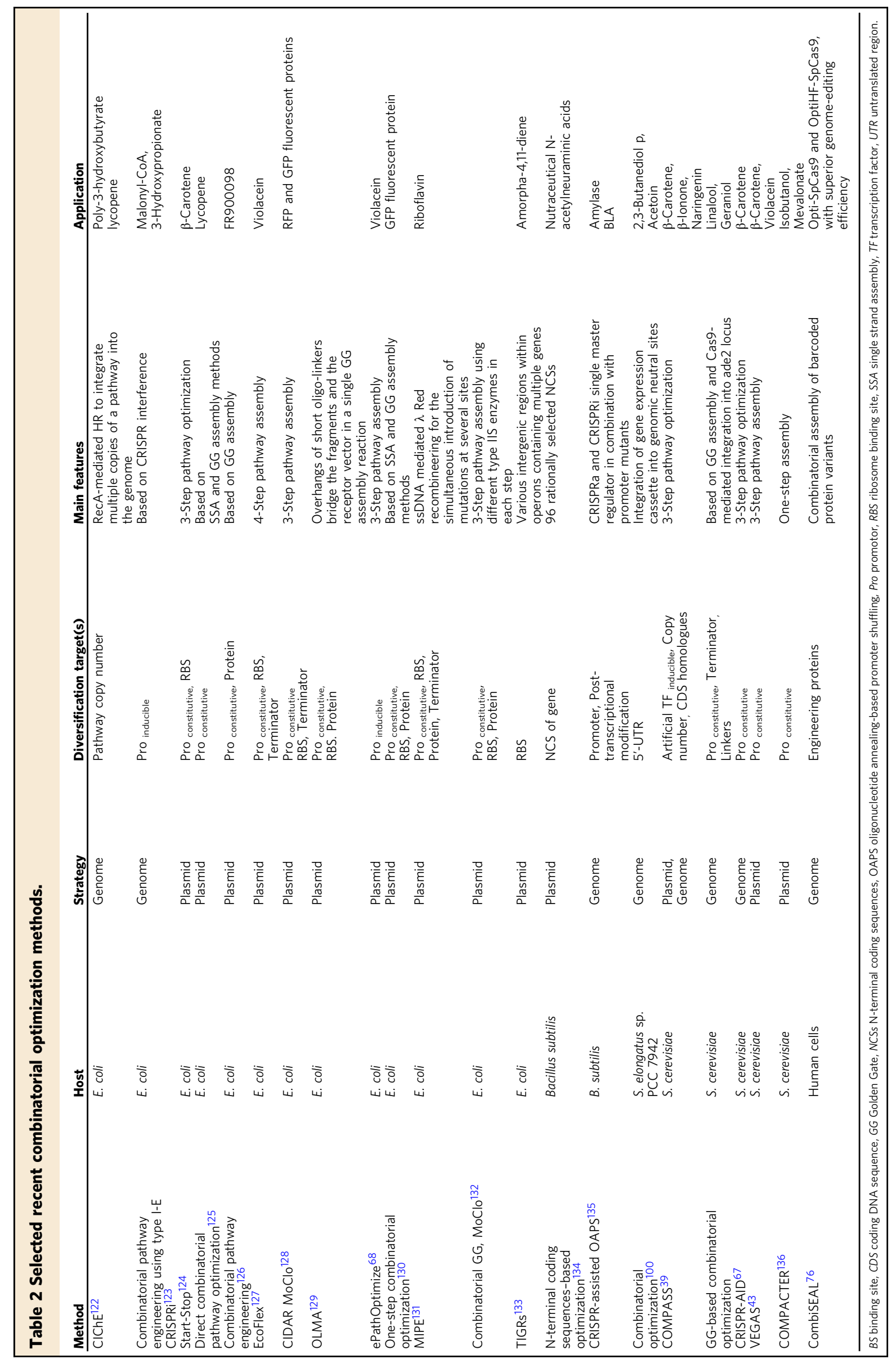


when predictable regulator behavior is required (as the genomic integration position affects the protein expression level $)^{39}$.

Up to this point, we have highlighted the power of combinatorial optimization in order to facilitate expressing heterologous pathways in an in vivo system. The in vitro system relies on designing functional biological systems from scratch. Recently a straightforward in vitro method to smartly generate DNA library construction has been developed called "in vitro SCRaMbLE system" (an abbreviation for Synthetic Chromosome Rearrangement and Modification by LoxP-mediated Evolution) enabling optimization of the biosynthetic pathway flux via rearranging relevant transcription units ${ }^{69}$. This system uses Cre recombinase and purified DNA encoding multiple loxPsym sites mixed in a tube. Wu et al. ${ }^{69}$ demonstrated two strategies using the "in vitro SCRaMbLE system" for pathway optimization: (i) The top-down method that consists of a purified DNA plasmid encoding multiple loxPsym sites. Addition of Cre recombinase to the reaction allows generating a library of SCRaMbLE DNA. (ii) The bottom-up system that employs an "acceptor vector" and a pool of "donor fragments" flanked by loxPsym sites. With the addition of Cre recombinase to the tube, donor fragments are randomly assembled into the acceptor vector. Therefore, a library of diverse plasmids is generated. The plasmid library can be subsequently transferred to a host strain for identification of genotype of each individual derivative. The in vitro SCRaMbLE system basically provides optimization in ways that the in vivo system cannot accomplish. However, application of combinatorial optimization strategies in in vitro production of proteins (cell-free protein synthesis (CFPS) $)^{70}$ or ex vivo (experimentation performed on tissue from an organism in an external environment) ${ }^{71}$ systems have not been studied yet. Such systems are typically used to produce toxic and/or large protein complexes, as there is no need to maintain cell viability. Combinatorial optimization strategies can be implemented to generate various combinations of genes and regulators in one cloning reaction cocktail. The in vivo and ex vivo systems require cellular lysate, transcription and translation factors ${ }^{72}$ to express different subunits of a large protein complex from the complex library of linear or plasmid DNA.

Profiling diversity in combinatorial library. Linking the diversity within a combinatorial library at the DNA level to the production level of various members of combinatorial library can guide the debugging process to achieve the desired performance. However, the ability to capitalize on the combinatorial library diversity is limited by the number of individuals that can be tracked and assessed. To ascertain the diversity present in a combinatorial library, sequencing analysis is often used. While the identification of DNA diversity in small library can be achieved by Sanger sequencing, next-generation sequencing (NGS) has opened new ways to tackle complexity quality assessment of DNA diversity in a large library. Nevertheless, NGS is not suitable for identifying and characterizing ATFs implemented in COMPASS $^{39}$ due to presence of repetitive sequences (e.g. ADs, minimal promoters, and terminators) in various ATFs.

Mapping the effects of protein mutation on its activity through employing high-throughput protein engineering methods or highly parallel mapping of genes to traits facilitated by multiplex DNA synthesis are now available and allow testing of a whole (or major fraction of) library population ${ }^{73}$. Microarray technology combined with molecular barcoding has been used to enable parallel tracking of genetically different individuals ${ }^{74}$. For example, trackable multiplex recombineering (TRMR) allows evaluation of thousands of definite genetic modifications in E. coli within a week. To do this, synthetic DNA cassettes with associated molecular barcodes were integrated into the $E$. coli genome, producing thousands of variants. Barcode sequences and microarrays were then used to compute population dynamics $^{42,75}$. In another example, a pooled library of barcoded labelled mutants of Streptococcus pyogenes Cas9 (SpCas9) nuclease were easily tracked by high-throughput short-read sequencing ${ }^{76}$.

We can extend the design principle of these methodologies to combinatorial libraries. Each DNA cassette encoding the individual fragment (e.g. regulator, gene of interest, terminator) can conceivably be labeled with a unique molecular barcode. Thereby, thousands of fragment combinations can be tracked by counting the frequency of the molecular barcodes. Moreover, sequencing short barcode fragments can significantly reduce the time and cost for sequencing regulators and downstream genes in individual isolates. However, to track the evolving genetic heterogeneity in a population of growing production cells, sequencing individual short barcode fragments is not sufficient and more advanced sequencing techniques such as "deep DNA sequencing" are required ${ }^{47}$. Deep sequencing, i.e. sequencing a genomic region multiple times, is an NGS approach that can be applied to track the genetic heterogeneity in a library of cell isolates. Höllerer et al. introduced a wildly applicable DNA-based phenotypic recording approach to generate huge datasets linking regulators to quantitative functional readouts of high precision, only relying on sequencing short tag DNA elements ${ }^{77}$. The technique implements a site-specific recombinase, a regulator that controls recombinase expression, and a DNA substrate modifiable by the recombinase. Both regulator sequence and substrate state can be determined in a single sequencing read, and the frequency of modified substrates amongst constructs harboring the same regulator presents the quantitative effect of regulator (transcriptional output of regulator) on recombinase expression. Using next-generation sequencing, the quantitative expression effect of large library of regulators can be quantified in parallel. As a proof of principle, this approach was applied to record translation kinetics of more than 300,000 bacterial RBSs, collecting over 2.7 million sequence-function pairs in a single experiment. However, resolution of real genetic diversity in such a population can be an issue. Very recently, Askary et al. presented Zombie system for image-based readout of DNA barcodes $^{75}$. In this system, phage RNA polymerases transcribe genomically integrated barcodes in fixed cells. The transcript RNA is then detected by fluorescent in situ hybridization. Singlenucleotide differences between barcodes are recognizable on the basis of the relative signal intensity of competing match and mismatch probes.

Identification of top producer in combinatorial library. Selection of strains with the highest product yield from a library with huge genetic diversity is a serious bottleneck. Formation of colored products that make microbial colonies screenable is an option. However, most chemicals are not colorful and their detection requires other methods. In that case, direct quantification of product titer using low-throughput gas or liquid chromatography analysis is typically used despite being timeconsuming. Methods based on spectroscopic enzymatic assay analytics are alternatives; however, they also have limited throughput. A more sophisticated solution is utilizing biosensor circuits to assay the target ligand at the single-cell level and translate their concentrations into more quantifiable signals. Biosensors are paired with high-throughput approaches, including flow cytometry and microfluidics, which allow isolation of variant cells with the phenotype of interest. 
a

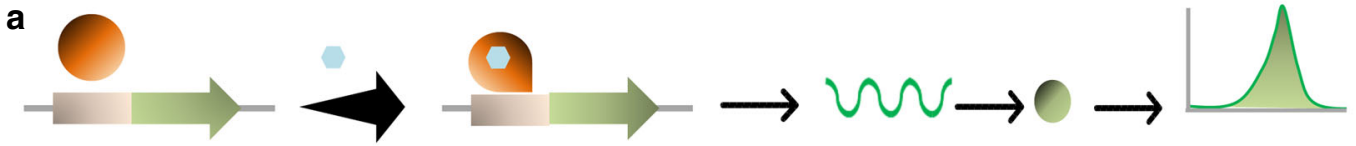

b

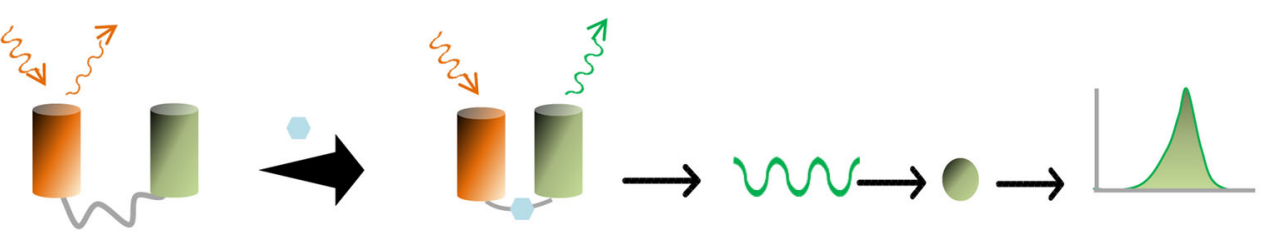

C

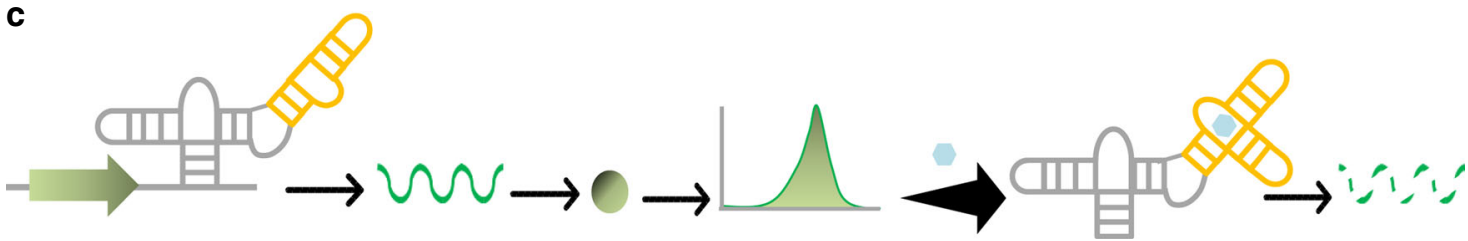

Fig. 4 Diverse biosensors used for screening combinatorial libraries. a The conformation of transcription factor (TF, orange oval) changes to active form upon binding the target ligand (blue octagon). When activated, the TF binds to its binding site (light orange square), upstream of a fluorescent reporter gene, to induce production of a fluorescent reporter protein (green oval) that is detected by flow cytometry. $\mathbf{b}$ FRET sensors comprised of a donor-acceptor fluorophore pair. Ligand is sandwiched between the two donor (orange cylinder) and acceptor fluorophores (green cylinder). Therefore, a conformation of FRET is changed that allows detecting the fluorescent signal by flow cytometry. c Correctly folded aptamer structure of riboswitch (orange-gray structure) allows transcription of fluorescent reporter gene (green arrow). The production of fluorescent protein (green oval) is detected by flow cytometry. In presence of ligand (blue octagon), the secondary structure of riboswitch device is changed. Consequently, transcription of its fluorescent reporter gene is inhibited.

Only TF-5,78,79 (Fig. 4a), fluorescence resonance energy transfer (FRET) ${ }^{-80}$ (Fig. 4b), and RNA-based biosensors- ${ }^{3,11,81-83}$ (Fig. 4c) based biosensors are commonly utilized for metabolic engineering. TF-based biosensors, natural sensory proteins that regulate gene expression in response to environmental signals, employ the host's transcription system to drive the expression of a reporter gene. Skjoedt et al. ${ }^{84}$ (2016) implemented systematic engineering of multiple parameters to establish a general biosensor design in the yeast $S$. cerevisiae based on metasbolite binding transcriptional activators from the superfamily of LysR-type transcriptional regulators (LTTRs) of prokaryotes. They next used the biosensors to screen cells producing different level of naringenin or cis, cismuconic acid. The designed biosensor output correlated with the production of metabolite. LTTR-based biosensors have already shown their utility to screen top producers: in one example they were used for screening $0.0000025 \%$ of the theoretical complexity of Narion ( $\beta$-ionone and naringenin co-producer) library. Rogers et al. attempted to evaluate the tuning of four genetically encoded protein sensors that respond to acrylate, glucarate, erythromycin and naringenin on either low-copy or high-copy plasmids 5 . Higher number of the intracellular gene constructs ensured higher dynamic range as well as rapid response to the target ligand ${ }^{5}$. However, TFbased biosensors typically have relatively high background noise, and a lot of current research focuses on addressing this shortcoming. FRET biosensors encompass a pair of donor and acceptor fluorophores. A ligand-binding peptide is sandwiched between a pair of donor and acceptor fluorophores, and ligand binding is observed via the FRET change. A set of FRET biosensors, based on three pairs of donor/acceptor, carboxyfluorescein (FAM)/Boron dipyrromethene (BODIPY) ${ }^{85}$, Nitrobenzoxadiazole (NBD)/nonsteroidal dye (Nile red) ${ }^{86}$, and coumarine/NBD ${ }^{86}$, was developed for real time monitoring of acid sphingomyelinase at high sensitivity and with high spatial resolution. The FRET biosensor is selectively cleaved by sphingomyelinase that leads to significant increase in fluorescence of the fluorescein FRET donor ${ }^{85}$. FRET biosensors have high orthogonality, high temporal resolution, and relatively easy construction ${ }^{80}$. The RNA-based biosensors include RNA riboswitches and RNA Spinaches. In the case of RNA riboswitchbased biosensors, the regulatory domain of an mRNA selectively binds to a ligand resulting in a structural change to the response domain that regulates translation of its encoded protein. Abatemarco et al. developed RNA riboswitch-based biosensor to detect the production of tyrosine and streptavidin concentrations in S. cerevisiae library. The aptamer is co-encapsulated with a member of a yeast library followed by incubation to produce the molecule of interest and development of a fluorescence signal. The picoliter droplets flow through a microfluidic device allowing sorting based on fluorescence ${ }^{87}$. In comparison to TF based biosensors, their kinetics are faster because the RNA has already been transcribed and so offer faster responses to target metabolite. They also do not rely on protein-protein or protein-metabolite interactions. This allows for more targeted engineering of the aptamers (the ligand binding domain) and the expression platforms. The development of biosensors that allow in vivo evaluation of any desired product of metabolic engineering would be an absolute boon for the field. A step in this direction is the systematic evolution of ligands by exponential enrichment (SELEX) approach, which can be, at least in principle, used to generate artificial riboswitch-based biosensors (single-stranded RNA aptamer) for any target metabolites. Using SELEX, metabolite-responsive riboswitch-based biosensors are developed from a library of nucleic acids. The selected riboswitch-based biosensors are, subsequently, employed to detect and report the metabolite signal ${ }^{88}$.

Very recently, a high-resolution methodology for the cell-specific RNA labeling was established based on nucleoside-enzyme pair ${ }^{89}$. The small molecule-enzyme pair consists of uridine/cytidine kinase 2 and $2^{\prime}$-azidouridine, where $2^{\prime}$-azidouridine is only incorporated in cells expressing uridine/cytidine kinase 2 . This pair can be used to purify and track RNA from specific cellular populations.

\section{Computational modeling to advance combinatorial optimization}

Metabolic engineering efforts aim to optimize the cellular processes for production of a compound of interest in host of choice 


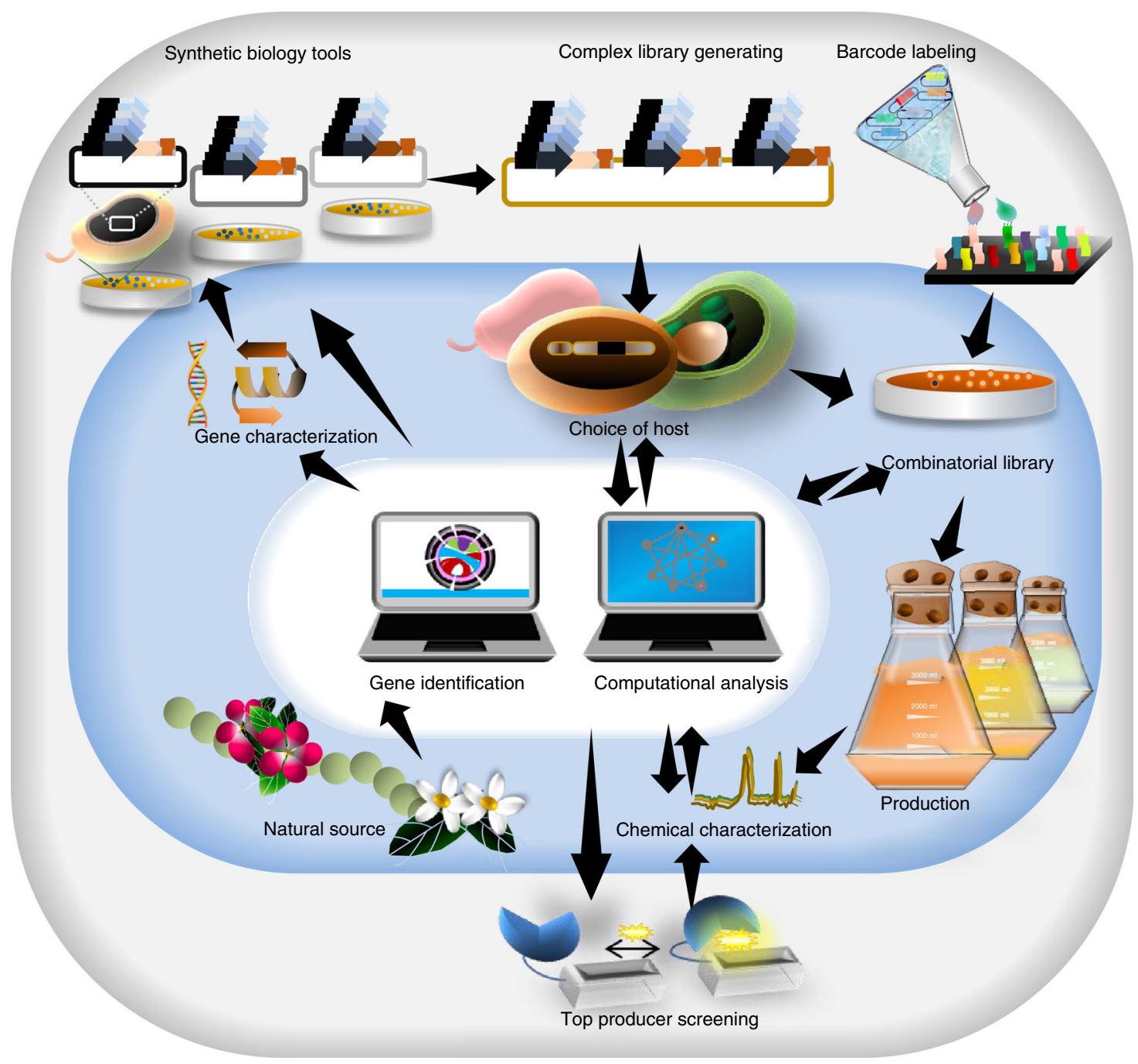

Fig. 5 Schematic overview of computational design and evaluation to achieve optimal performance. Synthetic biology tools are used to establish combinatorial optimization methods. The generated library is profiled using barcoding tools and biosensors allow to screen top producers within the library (gray area). Nature is a vital source of identified nutrients and pharmaceuticals. Metabolic engineering applies synthetic biology tools to produce compounds of the characterized biosynthetic pathway in a desired host (blue area). The production of certain compounds can be optimized using combinatorial optimization strategies. The data obtained from combinatorial library and its pre-characterized modules are computationally integrated to establish mathematical models to support the early design steps for chosen host on the basis of genome-scale metabolic modelling. The computational data suggest which synthetic pathways are the most promising in a given target organism and which host pathway genes need to be upregulated or be silenced based on knowledge of how different cellular subsystems work together. The best producers in combinatorial libraries can provide detailed information to feed into models that aim to uncover principles of how synthetic circuits behave in host systems. Blue arrows, regulators. Orange squares, CDSs. Brown "T", terminators.

(Fig. 5, blue area). To bridge actual and desired function of enzymes and pathways, the complexity of genome-wide metabolism necessitates directed evolution approaches ${ }^{90}$. The directed (or adaptive laboratory) evolution improves the natural evolution process by (i) randomization of mutation or recombination and (ii) accelerated screening rate of positive strains ${ }^{90}$. It uses different databases, libraries of tools and conditions to generate the optimal production rate of a desired compound. Mathematical models and computational simulation could be applied as powerful tools to understand and predict the behavior of the biological systems.

The multi-level omics data obtained from different databases for pre-characterized synthetic biology parts and modules are computationally integrated to establish mathematical models to support the early design steps of a system. The computational design (Fig. 5, white area) approaches allow connecting reactions obtained from various databases; for example, enzymes can often recognize molecules that are similar to their natural precursor. Next, computational design approaches calculate all possible metabolic pathways to achieve wanted product. Although the computational modeling of synthetic metabolic networks has improved tremendously, the experimental data obtained from modeled synthetic pathways is not always in agreement with the best modeled pathways suggested by computational modelling (in even very well characterized organisms). In fact, to generate data for computational model building, better high-throughput techniques for analysis of the cellular transcriptome, proteome, and metabolome are still necessary. Some combinations of pathways may include unrealistic reactions or too long pathways. Moreover, dynamic models usually do not cover a large number of reactions commonly involved in a pathway. In such a case, the counted pathways required to be evaluated. Recently, Carbonell et al. built and 
demonstrated an automated Design-Build-Test-Learn (DBTL) pipeline to computationally select the most promising parts, e.g. enzyme coding regions and RBSs, for assembly, allowing significantly more manageable experimental screening effort ${ }^{91}$. The DBTL pipeline led to a 500 -fold increase in titer of the flavonoid (2S)-pinocembrin from screening of only 65 variants out of more than 23,000 possible combinations. Although this system was developed for E. coli, in principle it should be applicable for synthesis in other chassis. RBS Calculator allows the prediction of the translation initiation rate of internal start codons, particularly to minimize undesired expression in $E$. coli ${ }^{19,92}$.

Several toolboxes are now available to integrate transcriptomics, proteomics, fluxomics and metabolomics data. TnCore allows automatic integration of Tn-Seq data in addition to RNA seq data to generate context specific models. This provides a "systems-level" view for metabolic engineering aims ${ }^{93}$. Principal component analysis (PCA) was recently implemented to evaluate various engineered strains for metabolites that contribute to acetol formation using metabolomics datasets. This data helped identify NADPH regeneration as the bottleneck for efficient acetol biosynthesis 94 . However, few laboratories work concurrently on both experimental and computational aspects of metabolic engineering, a fact that impedes the robust information exchange between these domains that is necessary for the highest productivity. RiboLogic tool was developed for designing riboswitches that are responsive to RNA inputs, as well as small molecule ligands ${ }^{3}$. RiboLogic algorithm was applied to design 286 switches that modulate MS2 (bacteriophage coat protein) binding of flavin mononucleotide, tryptophan, theophylline, and miR-208a, a 22-nt miRNA implicated in cardiac hypertrophythe.

The use of selective marker genes as biosensors, links the host production performance with growth rate. Therefore, biosensors make possible to study directed evolution of the microorganism or the entire biological network (Fig. 5, grey area). Additionally, combinatorial optimization, by rapid generating thousands to millions constructs, makes it possible to circumvent insufficient knowledge of in vivo reactions. This allows achieving the best compromise (solution) among conflicting pre-defined subjects. In other words, the best performer achieved from a combinatorial library can guide the debugging process to achieve the wanted performance. This data combined can be used to design a computational model for generation of optimal constructs through mathematical data to minimize or maximize wanted functions out of required subjects. Finally, computational methods can suggest possible synthetic pathways that are predicted to perform the best in a target organism and the performance of the chosen synthetic pathways is evaluated in the laboratory. Overall, results achieved from combinatorial optimization and integration of these data into available computational predictive models, can provide better understanding of the whole cellular system ${ }^{95}$. Höllerer et al., implemented next-generation sequencing to assess the quantitative expression effect of extremely large sets of RBSs ${ }^{77}$ . They expanded from these large-scale datasets using a novel deep learning approach that combines ensembling and uncertainty modelling to predict the function of untested RBSs with high accuracy. The data achieved from DNA-based phenotypic recording supports deep learning databases and, therefore, provides a major advance in our ability to predict quantitative function from genetic sequence.

\section{Future perspective of combinatorial optimization}

Combinatorial optimization techniques can be implemented to construct gene circuits that satisfy quantitative performance, something that has been a long-standing challenge in synthetic biology (Fig. 6).
In the past 30 years, a number of microbial processes have been developed for the production of such high-value chemicals including taxol ${ }^{33}$, strictosidine ${ }^{96}$, opioids ${ }^{97}$, and cocoa butter-like lipids ${ }^{98}$. Until now, production of only a few compounds, such as 1,3-propanediol $23,99,1$-4-butanediol ${ }^{26,100}, \beta$-farnesene ${ }^{45,101}$, and amorphadiene $^{102}$ has reached commercial scale. In order to achieve fermentation titres of 25 grams per litre of artemisinic acid, plant-derived heterologous genes were engineered into $S$. cerevisiae. Furthermore, an efficient and scalable chemical process for the conversion of artemisinic acid to artemisinin using a chemical source of singlet oxygen was developed ${ }^{102}$. In another study, the heterologous genes encoding biosynthetic enzymes of the taxol biosynthetic pathway, isoprenoid pathway, and the regulatory factors to inhibit competitive pathways were introduced into the yeast $S$. cerevisiae to produce 33 milligram per liter of taxadiene ${ }^{33}$. In both projects, the yeast promoters were used to express the engineered enzymes on a high-copy plasmid. Inducible orthogonal regulators 8 , and combinatorial optimization approaches allowing to genomically integration of pathways genes $^{67}$ can pave the way to producing natural and novel chemicals for new applications such as "non-natural" variants of thaxtomin phytotoxins ${ }^{73}$ and new peptides with "designer" functional properties. Protein production for biotechnological and medicinal applications is a multibillion-dollar market. Chemical synthesis of protein molecules is prohibitively expensive. Several expression systems ranging from bacterial hosts to mammalian cells have been established ${ }^{24}$. However, only 25 of them have been produced at a bioreactor scale ${ }^{24}$. In fact, biological production of complex proteins is facing a series of challenges, such as the need for balanced expression of multiple subunits of large proteins. Combinatorial optimization strategies allow expression of various subunits of protein complex in different ratios and, by doing so, facilitate the correct assembly of multi-subunit complexes (i.g. cytochromes, catalases, P450 enzymes, and carboxysomes).In particular, improvements in synthetic biology have made possible to study gene regulatory networks (GRNs) in a simplified setting amenable for exact experimental controls.

For example, researchers constructed mutual inhibition toggle between TetR and LacI inhibitors ${ }^{103,64}$, bistable circuit of autoactivation $^{104}$, or logic XOR and AND gates ${ }^{105}$. Combinatorial optimization coupled with biosensor techniques can facilitate generation of different possible GRNs, whose output can be measured and compared by placing a reporter gene downstream of the last regulator of the network and the output quantified by flow cytometry. This paves the way to understand regulatory relationships among genes and identifying key regulators and bottlenecks in GRNs.

There is no super host that is best for the production of all target molecules ${ }^{106}$. S. cerevisiae is a better production host than $E$. coli for production of amorphadiene, and $\beta$-farnesene due to less toxicity of pathway intermediates ${ }^{101,102}$, but producer populations were compromised by a high percentage of nonproducer mutants ${ }^{47}$. Hypothetical scenario to handle especially complex synthesis pathways is to divide them into pieces and design a different synthetic microbial host for each piece. The engineered microbes can then be grown together in consortia 107,108 . This can be substantially mitigated by utilizing combinatorial design principles to optimization multiple portions of the complex pathway (across multiple hosts) in parallel.

\section{Future directions}

Synthetic biology projects often need tunable expression of various combinations of genes to achieve an optimal output. Ideally, computational models will ultimately facilitate the forward- 


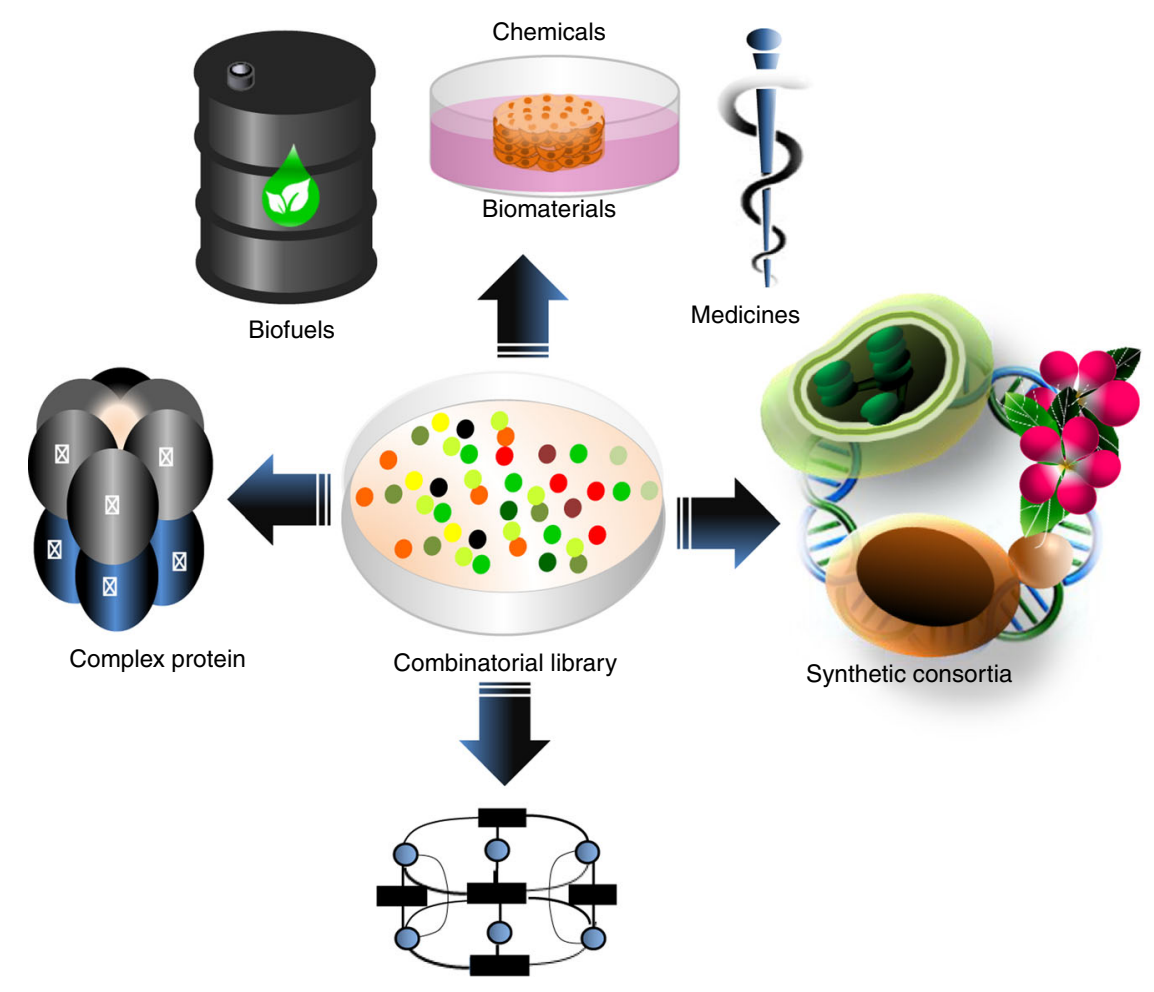

Gene regulatory network

Fig. 6 Application of combinatorial optimization strategies. One main aim of synthetic biology is development of microbial strains able to optimize and maximize yield and productivity of target chemicals, e.g. biofuels, biomaterials and, medicines, or multi-subunit cellular complexes that can be facilitated by applying combinatorial optimization approaches. Another interesting goal of synthetic biology is engineering sophisticated GRNs to expose the genetic architecture of complex traits and diseases. Smartly designed combinatorial libraries can generate huge number of GRN variants, where the optimal expression level of regulators of networks can be monitored. To overcome limitations regarding the transferability and expression of all involved systems in one chassis, a promising alternative solution is to focus on parallel optimization of metabolic pathways divided among different cells in synthetic microbial consortia107,108.

engineering of many parts to achieve desired function. However, until then the best alternative is to rapidly generate and accurately characterize as many new variants as possible. Therefore, smartly designed combinatorial optimization methods for the empirical balancing of metabolic pathway gene expression is of interest to a broad range of synthetic biologists. The workflow described in this review has the potential to become an enabling standard for researchers seeking to achieve optimal output while minimizing experimental effort in bioengineering projects. We expect that future developments in combinatorial optimization will still be limited by a lack of tools to screen the best producers from such a library. To continue advancing in our ability to engineer living organisms for high-level production of desired compounds using combinatorial optimization methods, we will require highthroughput screening techniques. Therefore, an interesting research direction would be a generalized biosensor construction framework for targeting diverse molecules. Ongoing advancements in developing riboswitch-based biosensors may become a larger part of the solution.

In this review, we discussed how DNA barcoding methodologies have significantly improved in recent years, enabling the computation of population dynamics ${ }^{42,75}$ or tracking of mutants of Cas9 nuclease library ${ }^{76}$. Such techniques however have yet to be applied for tracking of combinatorial library members. In fact, we can envision the use of the design principle of these methodologies to combinatorial libraries to identify the DNA elements combined in libraries of million circuits.

Combinatorial optimization in synthetic biology encompasses a number of different techniques and disciplines that include molecular biology, analytical and protein chemistry and computational modelling. Progress in this field is emerging from a close interplay between bioinformaticians, chemists, and synthetic biologists.

Received: 26 January 2019; Accepted: 15 April 2020;

Published online: 15 May 2020

\section{References}

1. Silva-Rocha, R. \& de Lorenzo, V. Mining logic gates in prokaryotic transcriptional regulation networks. FEBS Lett. 582, 1237-1244 (2008).

2. Harbaugh, S. V., Goodson, M. S., Dillon, K., Zabarnick, S. \& KelleyLoughnane, N. Riboswitch-based reversible dual color sensor. ACS Synth. Biol. 6, 766-781 (2017).

3. Wu, M. J., Andreasson, J. O. L., Kladwang, W., Greenleaf, W. \& Das, R. Automated design of diverse stand-alone riboswitches. ACS Synth. Biol. 8, 1838-1846 (2019)

4. Tomazou, M., Barahona, M., Polizzi, K. M. \& Stan, G. B. Computational redesign of synthetic genetic oscillators for independent amplitude and frequency modulation. Cell Syst. 6, 508-520 (2018). e505.

5. Rogers, J. K. et al. Synthetic biosensors for precise gene control and real-time monitoring of metabolites. Nucleic Acids Res. 43, 7648-7660 (2015).

6. Lechner, A., Brunk, E. \& Keasling, J. D. The need for integrated approaches in metabolic engineering. Cold Spring Harb. Perspect. Biol. 8, a023903 (2016)

7. Jeschek, M., Gerngross, D. \& Panke, S. Combinatorial pathway optimization for streamlined metabolic engineering. Curr. Opin. Biotechnol. 47, 142-151 (2017).

8. Naseri, G. et al. Plant-derived transcription factors for orthologous regulation of gene expression in the yeast Saccharomyces cerevisiae. ACS Synth. Biol. 6, 1742-1756 (2017). 
9. Lebar, T. \& Jerala, R. Benchmarking of TALE- and CRISPR/dCas9-based transcriptional regulators in mammalian cells for the construction of synthetic genetic circuits. ACS Synth. Biol. 5, 1050-1058 (2016).

10. Alper, H. S. \& Avalos, J. L. Metabolic pathway engineering. Synth. Syst. Biotechnol. 3, 1-2 (2018).

11. Zhang, J., Jensen, M. K. \& Keasling, J. D. Development of biosensors and their application in metabolic engineering. Curr. Opin. Chem. Biol. 28, 1-8 (2015).

12. Mundt, M., Anders, A., Murray, S. M. \& Sourjik, V. A system for gene expression noise control in yeast. ACS Synth. Biol. 7, 2618-2626 (2018).

13. Ding, M. Z. et al. Biosynthesis of Taxadiene in Saccharomyces cerevisiae: selection of geranylgeranyl diphosphate synthase directed by a computeraided docking strategy. PLoS ONE 9, e109348 (2014).

14. Mariño-Ramírez, L., Kann, M. G., Shoemaker, B. A. \& Landsman, D. Histone structure and nucleosome stability. Expert Rev. Proteom. 2, 719-729 (2005).

15. Machens, F., Balazadeh, S., Mueller-Roeber, B. \& Messerschmidt, K. Synthetic promoters and transcription factors for heterologous protein expression in Saccharomyces cerevisiae. Front Bioeng. Biotechnol. 5, 63 (2017).

16. Chen, Y. J. et al. Characterization of 582 natural and synthetic terminators and quantification of their design constraints. Nat. Methods 10, 659-664 (2013)

17. Curran, K. A. et al. Short synthetic terminators for improved heterologous gene expression in yeast. ACS Synth. Biol. 4, 824-832 (2015).

18. Zampini, M. et al. Terminator operon reporter: combining a transcription termination switch with reporter technology for improved gene synthesis and synthetic biology applications. Sci. Rep. 6, 26572 (2016).

19. Salis, H. M., Mirsky, E. A. \& Voigt, C. A. Automated design of synthetic ribosome binding sites to control protein expression. Nat. Biotechnol. 27, 946-950 (2009). The authors developed a biophysical models for designing synthetic ribosome binding sites that predict identical ribosome binding site sequences in different genetic contexts can result in different protein expression levels.

20. Lewis, J. C. Metallopeptide catalysts and artificial metalloenzymes containing unnatural amino acids. Curr. Opin. Chem. Biol. 25, 27-35 (2015).

21. Werner, N., Ramirez-sarmiento, C. A. \& Agosin, E. Protein engineering of carotenoid cleavage dioxygenases to optimize $\beta$-ionone biosynthesis in yeast cell factories. Food Chem. 299, 125089 (2019).

22. Lopez, J. et al. Production of beta-ionone by combined expression of carotenogenic and plant CCD1 genes in Saccharomyces cerevisiae. Micro. Cell Fact. 14, 84 (2015)

23. King, Z. A. \& Feist, A. M. Optimal cofactor swapping can increase the theoretical yield for chemical production in Escherichia coli and Saccharomyces cerevisiae. Metab. Eng. 24, 117-128 (2014).

24. Vandermies, M. \& Fickers, P. Bioreactor-scale strategies for the production of recombinant protein in the yeast Yarrowia lipolytica. Microorganisms 7, 40 (2019).

25. Teixeira, P. G., David, F., Siewers, V. \& Nielsen, J. Engineering lipid droplet assembly mechanisms for improved triacylglycerol accumulation in Saccharomyces cerevisiae. FEMS Yeast Res. 18, foy060 (2018).

26. Liu, H. \& Lu, T. Autonomous production of 1,4-butanediol via a de novo biosynthesis pathway in engineered Escherichia coli. Metab. Eng. 29, 135-141 (2015).

27. Chen, X. \& Zhang, J. The genomic landscape of position effects on protein expression level and noise in yeast. Cell Syst. 2, 347-354 (2016).

28. Xie, W., Ye, L., Lv, X., Xu, H. \& Yu, H. Sequential control of biosynthetic pathways for balanced utilization of metabolic intermediates in Saccharomyces cerevisiae. Metab. Eng. 28, 8-18 (2015).

29. Scalcinati, G. et al. Combined metabolic engineering of precursor and cofactor supply to increase alpha-santalene production by Saccharomyces cerevisiae. Micro. Cell Fact. 11, 117 (2012).

30. Sander, T., Wang, C. Y., Glatter, T. \& Link, H. CRISPRi-based downregulation of transcriptional feedback improves growth and metabolism of arginine overproducing E. coli. ACS Synth. Biol. 8, 1983-1990 (2019).

31. Garcia-Granados, R., Lerma-Escalera, J. A. \& Morones-Ramirez, J. R. Metabolic engineering and synthetic biology: synergies, future, and challenges. Front. Bioeng. Biotechnol. 7, 36 (2019).

32. Cambray, G., Guimaraes, J. C. \& Arkin, A. P. Evaluation of 244,000 synthetic sequences reveals design principles to optimize translation in Escherichia coli. Nat. Biotechnol. 36, 1005-1015 (2018).

33. Engels, B., Dahm, P. \& Jennewein, S. Metabolic engineering of taxadiene biosynthesis in yeast as a first step towards Taxol (Paclitaxel) production. Metab. Eng. 10, 201-206 (2008).

34. Smanski, M. J. et al. Functional optimization of gene clusters by combinatorial design and assembly. Nat. Biotechnol. 32, 1241-1249 (2014).

35. Alper, H. \& Stephanopoulos, G. Global transcription machinery engineering: a new approach for improving cellular phenotype. Metab. Eng. 9, 258-267 (2007).

36. Warnecke, T. E. et al. Rapid dissection of a complex phenotype through genomic-scale mapping of fitness altering genes. Metab. Eng. 12, 241-250 (2010).
37. Nyerges, A. et al. A highly precise and portable genome engineering method allows comparison of mutational effects across bacterial species. Proc. Natl Acad. Sci. USA 113, 2502-2507 (2016).

38. Wang, H. H. et al. Programming cells by multiplex genome engineering and accelerated evolution. Nature 460, 894-898 (2009).

39. Naseri, G., Behrend, J., Rieper, L. \& Mueller-Roeber, B. COMPASS for rapid combinatorial optimization of biochemical pathways based on artificial transcription factors. Nat. Commun. 10, 2615 (2019).

40. Lim, H. G., Jang, S., Jang, S., Seo, S. W. \& Jung, G. Y. Design and optimization of genetically encoded biosensors for high-throughput screening of chemicals. Curr. Opin. Biotechnol. 54, 18-25 (2018).

41. Lee, H.-M., Vo, P. \& Na, D. Advancement of metabolic engineering assisted by synthetic biology. Catalysts. 8, 619 (2018).

42. Roy, K. R. et al. Multiplexed precision genome editing with trackable genomic barcodes in yeast. Nat. Biotechnol. 36, 512-520 (2018).

43. Mitchell, L. A. et al. Versatile genetic assembly system (VEGAS) to assemble pathways for expression in S. cerevisiae. Nucleic Acids Res. 43, 6620-6630 (2015).

44. Zhang, J., Feng, T., Wang, J., Wang, Y. \& Zhang, X. H. The mechanisms and applications of quorum sensing (QS) and quorum quenching (QQ). J. Ocean Univ. China 18, 1427-1442 (2019).

45. Nakamura, M. et al. Anti-CRISPR-mediated control of gene editing and synthetic circuits in eukaryotic cells. Nat. Commun. 10, 194 (2019). Various cells types that were pre-engineered with a set of anti-CRISPR molecules become resistant to gene editing, thus providing a means to generate "writeprotected" cells that prevent future gene editing.

46. Sandoval, C. M. et al. Use of pantothenate as a metabolic switch increases the genetic stability of farnesene producing Saccharomyces cerevisiae. Metab. Eng. 25, 215-226 (2014)

47. Neilson, J. R. \& Sharp, P. A. Small RNA regulators of gene expression. Cell 134, 899-902 (2008).

48. Rugbjerg, P. \& Sommer, M. O. A. Overcoming genetic heterogeneity in industrial fermentations. Nat. Biotechnol. 37, 869-876 (2019).

49. Wilson, K. A., Chateau, M. L. \& Porteus, M. H. Design and development of artificial zinc finger transcription factors and zinc finger nucleases to the hTERT locus. Mol. Ther. Nucleic Acids 2, e87 (2013).

50. Rantasalo, A., Kuivanen, J., Penttilä, M., Jäntti, J. \& Mojzita, D. Synthetic Toolkit for Complex Genetic Circuit Engineering in Saccharomyces cerevisiae. ACS Synthetic. Biology 7, 1573-1587 (2018).

51. Kleinjan, D. A., Wardrope, C., Nga Sou, S. \& Rosser, S. J. Drug-tunable multidimensional synthetic gene control using inducible degron-tagged dCas9 effectors. Nat. Commun. 8, 1191 (2017).

52. Chatelle, C. et al. A green-light-Responsive system for the control of transgene expression in mammalian and plant cells. ACS Synth. Biol. 7, 1349-1358 (2018).

53. Hochrein, L., Machens, F., Messerschmidt, K. \& Mueller-Roeber, B. PhiReX: a programmable and red light-regulated protein expression switch for yeast. Nucleic Acids Res. 45, 9193-9205 (2017)

54. Pathak, G. P., Vrana, J. D. \& Tucker, C. L. Optogenetic control of cell function using engineered photoreceptors. Biol. Cell 105, 59-72 (2013).

55. Courtney, T. M., Horst, T. J., Hankinson, C. P. \& Deiters, A. Synthesis and application of light-switchable arylazopyrazole rapamycin analogs. Org. Biomol. Chem. 17, 8348-8353 (2019).

56. Pushkarev, A. et al. A distinct abundant group of microbial rhodopsins discovered using functional metagenomics. Nature 558, 595-599 (2018).

57. Hochrein, L., Mitchell, L. A., Schulz, K., Messerschmidt, K. \& Mueller-Roeber, B. L-SCRaMbLE as a tool for light-controlled Cre-mediated recombination in yeast. Nat. Commun. 9, 1931 (2018).

58. Naseri, G., Behrend, J., Rieper, L. \& Mueller-Roeber, B. COMPASS for rapid combinatorial optimization of biochemical pathways based on artificial transcription factors. Nat. Commun. 10, 2615 (2019).

59. Bao, Z. et al. Homology-integrated CRISPR-Cas (HI-CRISPR) system for onestep multigene disruption in Saccharomyces cerevisiae. ACS Synth. Biol. 4, 585-594 (2015)

60. Legut, M. et al. High-throughput screens of PAM-flexible Cas9 variants for gene knock-out and transcriptional modulation. Cell Rep. 30, 2859-2868 (2020).

61. Teng, F. et al. Enhanced mammalian genome editing by new Cas12a orthologs with optimized crRNA scaffolds. Genome Biol. 20, 15 (2019).

62. Xu, T., Li, Y., Van Nostrand, J. D., He, Z. \& Zhou, J. Cas9-based tools for targeted genome editing and transcriptional control. Appl Environ. Microbiol. 80, 1544-1552 (2014)

63. Yu, Y. et al. An efficient gene knock-in strategy using 5'-modified doublestranded DNA donors with short homology arms. Nat. Chem. Biol. 16, 387-390 (2019).

64. Yeung, E. et al. Biophysical constraints arising from compositional context in synthetic gene networks. Cell Syst. 5, 11-24 (2017). 
65. Sheets, M. B., Wong, W. W. \& Dunlop, M. J. Light-inducible recombinases for bacterial optogenetics. ACS Synth. Biol. 9, 227-235 (2019).

66. Barbieri, E. M., Muir, P., Akhuetie-Oni, B. O., Yellman, C. M. \& Isaacs, F. J. Precise editing at dna replication forks enables multiplex genome engineering in eukaryotes. Cell 171, 1453-1467 (2017). e1413.

67. Lian, J., HamediRad, M., Hu, S. \& Zhao, H. Combinatorial metabolic engineering using an orthogonal tri-functional CRISPR system. Nat. Commun. 8, 1688 (2017).

68. Jones, J. A. et al. ePathOptimize: a combinatorial approach for transcriptional balancing of metabolic pathways. Sci. Rep. 5, 11301 (2015).

69. Wu, Y. et al. In vitro DNA SCRaMbLE. Nat. Commun. 9, 1935 (2018).

70. Lo Gullo, G. et al. Optimization of an in vitro transcription/translation system based on sulfolobus solfataricus cell lysate. Archaea 2019, 9848253 (2019).

71. Misra, S. et al. Ex vivo organotypic culture system of precision-cut slices of human pancreatic ductal adenocarcinoma. Sci. Rep. 9, 2133 (2019). The authors developed a optimized culture conditions to keep tissue from human pancreas and pancreatic cancer viable for at least 4 days allowing ex vivo testing.

72. Khambhati, K. et al. Exploring the potential of cell-free protein synthesis for extending the abilities of biological systems. Front Bioeng. Biotechnol. 7, 248 (2019).

73. Winn, M., Francis, D. \& Micklefield, J. De novo biosynthesis of "non-natural" thaxtomin phytotoxins. Angew. Chem. Int Ed. Engl. 57, 6830-6833 (2018).

74. Warner, J. R., Reeder, P. J., Karimpour-Fard, A., Woodruff, L. B. \& Gill, R. T. Rapid profiling of a microbial genome using mixtures of barcoded oligonucleotides. Nat. Biotechnol. 28, 856-862 (2010).

75. Askary, A. et al. In situ readout of DNA barcodes and single base edits facilitated by in vitro transcription. Nat. Biotechnol. 38, 66-75 (2020).

76. Choi, G. C. G. et al. Combinatorial mutagenesis en masse optimizes the genome editing activities of SpCas9. Nat. Methods. 16, 722-730 (2019). Authors presented CombiSEAL platform that enables scalable assembly and systematically characterizing library of barcoded protein variants.

77. Höllerer, S. et al. Large-scale DNA-based phenotypic recording and deep learning enable highly accurate sequence-function mapping. Preprint available at: https://www.biorxiv.org/content/10.1101/2020.01.23.915405v1.full (2020).

78. Wan, X., Marsafari, M. \& Xu, P. Engineering metabolite-responsive transcriptional factors to sense small molecules in eukaryotes: current state and perspectives. Micro. Cell Fact. 18, 61 (2019).

79. Juarez, J. F., Lecube-Azpeitia, B., Brown, S. L., Johnston, C. D. \& Church, G. M. Biosensor libraries harness large classes of binding domains for construction of allosteric transcriptional regulators. Nat. Commun. 9, 3101 (2018).

80. Piatkevich, K. D. \& Verkhusha, V. V. Guide to red fluorescent proteins and biosensors for flow cytometry. Methods Cell Biol. 102, 431-461 (2011). The authors chracterized the advanced orange, red, and far-red fluorescent proteins with the properties specifically required for the flow cytometry applications.

81. Santos-Merino, M., Singh, A. K. \& Ducat, D. C. New applications of synthetic biology tools for cyanobacterial metabolic engineering. Front Bioeng. Biotechnol. 7, 33 (2019).

82. Xiu, Y. et al. Naringenin-responsive riboswitch-based fluorescent biosensor module for Escherichia coli co-cultures. Biotechnol. Bioeng. 114, 2235-2244 (2017).

83. Wang, R. et al. Design and characterization of biosensors for the screening of modular assembled naringenin biosynthetic library in Saccharomyces cerevisiae. ACS Synth. Biol. 8, 2121-2130 (2019).

84. Skjoedt, M. L. et al. Engineering prokaryotic transcriptional activators as metabolite biosensors in yeast. Nat. Chem. Biol. 12, 951-958 (2016).

85. Kappe, C., Mohamed, Z. H., Naser, E., Carpinteiro, A. \& Arenz, C. A novel visible range FRET probe for monitoring acid sphingomyelinase activity in living cells. Chem. Eur. https://doi.org/10.1002/chem.202000133 (2020).

86. Pinkert, T., Furkert, D., Korte, T., Herrmann, A. \& Arenz, C. Amplification of a FRET probe by lipid-water partition for the detection of acid sphingomyelinase in live cells. Angew. Chem. Int Ed. Engl. 56, 2790-2794 (2017).

87. Abatemarco, J.et al. RNA-aptamers-in-droplets (RAPID) high-throughput screening for secretory phenotypes. Nat. Commun. 8, 332 (2017). A RNAaptamers-in-droplets method, called RAPID, was developed that employs the generality of ultrahigh-throughput microfluidic and aptamer technologies to transduce extracellular product titer into fluorescence to screen millions of variants.

88. Carpenter, A. C., Paulsen, I. T. \& Williams, T. C. Blueprints for biosensors: design, limitations, and applications. Genes (Basel) 9, E375 (2018).

89. Nainar, S. et al. An optimized chemical-genetic method for cell-specific metabolic labeling of RNA. Nat. Methods 17, 311-318 (2020). A small molecule-enzyme pair consisting of uridine/cytidine kinase 2 and $2^{\prime}$ azidouridine was developed for labeling cells expressing uridine/cytidine kinase 2 using $2^{\prime}$-azidouridine.
90. Zhou, S. \& Alper, H. S. Strategies for directed and adapted evolution as part of microbial strain engineering. J. Chem. Technol. Biotechnol. 94, 366-376 (2019).

91. Carbonell, P. et al. An automated Design-Build-Test-Learn pipeline for enhanced microbial production of fine chemicals. Commun. Biol. 1, 66 (2018).

92. Salis, H. M. The ribosome binding site calculator. Methods Enzymol. 498, 19-42 (2011).

93. diCenzo, G. C., Mengoni, A. \& Fondi, M. Tn-Core: a toolbox for integrating tn-seq gene essentiality data and constraint-based metabolic modeling. ACS Synth. Biol. 8, 158-169 (2019).

94. Ohtake, T. et al. Metabolomics-driven approach to solving a CoA imbalance for improved 1-butanol production in Escherichia coli. Metab. Eng. 41, 135-143 (2017). The authors reported the improvement of 1-butanol production in E. coli by resolving CoA imbalance using metabolome analysis coupled with rational metabolic engineering approaches.

95. Yan Chen, D. B., Mukhopadhyay, A., Petzold, C. J. Systems and synthetic biology tools for advanced bioproduction hosts. Curr. Opin. Biotechnology. 64, 101-109 (2020).

96. Brown, S., Clastre, M., Courdavault, V. \& O'Connor, S. E. De novo production of the plant-derived alkaloid strictosidine in yeast. Proc. Natl Acad. Sci. USA 112, 3205-3210 (2015).

97. Thodey, K., Galanie, S. \& Smolke, C. D. A microbial biomanufacturing platform for natural and semisynthetic opioids. Nat. Chem. Biol. 10, 837-844 (2014).

98. Bergenholm, D., Gossing, M., Wei, Y., Siewers, V. \& Nielsen, J. Modulation of saturation and chain length of fatty acids in Saccharomyces cerevisiae for production of cocoa butter-like lipids. Biotechnol. Bioeng. 115, 932-942 (2018).

99. Zhong, W., Zhang, Y., Wu, W., Liu, D. \& Chen, Z. Metabolic engineering of a homoserine-derived non-natural pathway for the de novo production of 1,3Propanediol from glucose. ACS Synth. Biol. 8, 587-595 (2019).

100. Oliver, J. W., Machado, I. M., Yoneda, H. \& Atsumi, S. Combinatorial optimization of cyanobacterial 2,3-butanediol production. Metab. Eng. 22, 76-82 (2014).

101. Leavell, M. D., McPhee, D. J. \& Paddon, C. J. Developing fermentative terpenoid production for commercial usage. Curr. Opin. Biotechnol. 37, 114-119 (2016)

102. Paddon, C. J. et al. High-level semi-synthetic production of the potent antimalarial artemisinin. Nature 496, 528-532 (2013).

103. Dimas, R. P. et al. Engineering DNA recognition and allosteric response properties of TetR family proteins by using a module-swapping strategy. Nucleic Acids Res. 47, 8913-8925 (2019).

104. Hermsen, R., Erickson, D. W. \& Hwa, T. Speed, sensitivity, and bistability in auto-activating signaling circuits. PLoS Comput. Biol. 7, e1002265 (2011).

105. Tamsir, A., Tabor, J. J. \& Voigt, C. A. Robust multicellular computing using genetically encoded NOR gates and chemical 'wires'. Nature 469, 212-215 (2011).

106. Fisher, A. K., Freedman, B. G., Bevan, D. R. \& Senger, R. S. A review of metabolic and enzymatic engineering strategies for designing and optimizing performance of microbial cell factories. Comput Struct. Biotechnol. J. 11, 91-99 (2014).

107. Jawed, K., Yazdani, S. S. \& Koffas, M. A. Advances in the development and application of microbial consortia for metabolic engineering. Metab. Eng. Commun. 9, e00095 (2019).

108. Wang, R., Zhao, S., Wang, Z. \& Koffas, M. A. Recent advances in modular coculture engineering for synthesis of natural products. Curr. Opin. Biotechnol. 62, 65-71 (2019).

109. Polstein, L. R. \& Gersbach, C. A. Light-inducible spatiotemporal control of gene activation by customizable zinc finger transcription factors. J. Am. Chem. Soc. 134, 16480-16483 (2012).

110. Konermann, S. et al. Optical control of mammalian endogenous transcription and epigenetic states. Nature 500, 472-476 (2013).

111. Müller, K. et al. A red/far-red light-responsive bi-stable toggle switch to control gene expression in mammalian cells. Nucleic Acids Res. 41, e77-e77 (2013).

112. Muller, K. et al. A red light-controlled synthetic gene expression switch for plant systems. Mol. Biosyst. 10, 1679-1688 (2014).

113. Polstein, L. R. \& Gersbach, C. A. A light-inducible CRISPR-Cas9 system for control of endogenous gene activation. Nat. Chem. Biol. 11, 198-200 (2015).

114. Nihongaki, Y., Yamamoto, S., Kawano, F., Suzuki, H. \& Sato, M. CRISPR Cas9-based photoactivatable transcription system. Chem. Biol. 22, 169-174 (2015).

115. Taslimi, A. et al. Optimized second-generation CRY2-CIB dimerizers and photoactivatable Cre recombinase. Nat. Chem. Biol. 12, 425-430 (2016).

116. Baumschlager, A., Aoki, S. K. \& Khammash, M. Dynamic blue light-inducible T7 RNA polymerases (Opto-T7RNAPs) for precise spatiotemporal gene expression control. ACS Synth. Biol. 6, 2157-2167 (2017). 
117. Xu, X. et al. A Single-component optogenetic system allows stringent switch of gene expression in yeast cells. ACS Synth. Biol. 7, 2045-2053 (2018).

118. Salinas, F. et al. Fungal light-oxygen-voltage domains for optogenetic control of gene expression and flocculation in yeast. mBio 9, e00626-18 (2018).

119. Yamada, M., Suzuki, Y., Nagasaki, S. C., Okuno, H. \& Imayoshi, I. Light control of the tet gene expression system in mammalian cells. Cell Rep. 25, e486 (2018).

120. An-adirekkun, J. et al. A yeast optogenetic toolkit (yOTK) for gene expression control in Saccharomyces cerevisiae. Biotechnol. Bioeng. 17, 886-893 (2020).

121. Geller, S. H., Antwi, E. B., Di Ventura, B. \& McClean, M. N. Optogenetic repressors of gene expression in yeasts using light-controlled nuclear localization. Cell. Mol. Bioeng. 12, 511-528 (2019). By using light to control the nuclear localization dead Cas9 protein, the expression of gene was regulated in yeast Saccharomyces cerevisiae cell.

122. Love, A. M., Biggs, B. W., Tyo, K. E. J. \& Ajikumar, P. K. Chemically inducible chromosomal evolution (ciche) for multicopy metabolic pathway engineering. Methods Mol. Biol. 1927, 37-45 (2019).

123. Tarasava, K., Liu, R., Garst, A. \& Gill, R. T. Combinatorial pathway engineering using type I-E CRISPR interference. Biotechnol. Bioeng. 115, 1878-1883 (2018).

124. Taylor, G. M., Mordaka, P. M. \& Heap, J. T. Start-Stop Assembly: a functionally scarless DNA assembly system optimized for metabolic engineering. Nucleic Acids Res. 47, e17 (2018).

125. Coussement, P., Bauwens, D., Maertens, J. \& De Mey, M. Direct combinatorial pathway optimization. ACS Synth. Biol. 6, 224-232 (2017).

126. Freestone, T. S. \& Zhao, H. Combinatorial pathway engineering for optimized production of the anti-malarial FR900098. Biotechnol. Bioeng. 113, 384-392 (2016).

127. Moore, S. J. et al. EcoFlex: a multifunctional MoClo kit for E. coli synthetic biology. ACS Synth. Biol. 5, 1059-1069 (2016).

128. Iverson, S. V., Haddock, T. L., Beal, J. \& Densmore, D. M. CIDAR MoClo: Improved MoClo assembly standard and new E. coli part library enable rapid combinatorial design for synthetic and traditional biology. ACS Synth. Biol. 5, 99-103 (2016).

129. Zhang, S., Zhao, X., Tao, Y. \& Lou, C. A novel approach for metabolic pathway optimization: Oligo-linker mediated assembly (OLMA) method. J. Biol. Eng. 9, 23 (2015).

130. Coussement, P., Maertens, J., Beauprez, J., Van Bellegem, W. \& De Mey, M. One step DNA assembly for combinatorial metabolic engineering. Metab. Eng. 23, 70-77 (2014).

131. Li, Y. et al. Multiplex iterative plasmid engineering for combinatorial optimization of metabolic pathways and diversification of protein coding sequences. ACS Synth. Biol. 2, 651-661 (2013).

132. Werner, S., Engler, C., Weber, E., Gruetzner, R. \& Marillonnet, S. Fast track assembly of multigene constructs using Golden Gate cloning and the MoClo system. Bioeng. Bugs 3, 38-43 (2012).

133. Pfleger, B. F., Pitera, D. J., Smolke, C. D. \& Keasling, J. D. Combinatorial engineering of intergenic regions in operons tunes expression of multiple genes. Nat. Biotechnol. 24, 1027-1032 (2006).
134. Tian, R. et al. Synthetic N-terminal coding sequences for fine-tuning gene expression and metabolic engineering in Bacillus subtilis. Metab. Eng. 55, 131-141 (2019).

135. Lu, Z. et al. CRISPR-assisted multi-dimensional regulation for fine-tuning gene expression in Bacillus subtilis. Nucleic Acids Res. 47, e40 (2019).

136. Du, J., Yuan, Y., Si, T., Lian, J. \& Zhao, H. Customized optimization of metabolic pathways by combinatorial transcriptional engineering. Nucleic Acids Res. 40, e142 (2012).

\section{Acknowledgements}

We thank Christoph Arenz for constructive feedback on the manuscript. Mattheos Koffas would like to acknowledge support from the National Science Foundation, Award Number MCB-1817631.

\section{Author contributions}

G.N. conceived and developed the review. G.N. and M.K. wrote the paper.

\section{Competing interests}

The authors declare no competing interests.

\section{Additional information}

Correspondence and requests for materials should be addressed to G.N. or M.A.G.K.

Peer review information Nature Communications thanks the anonymous reviewer(s) for their contribution to the peer review of this work.

Reprints and permission information is available at http://www.nature.com/reprints

Publisher's note Springer Nature remains neutral with regard to jurisdictional claims in published maps and institutional affiliations.

(c) (i) Open Access This article is licensed under a Creative Commons Attribution 4.0 International License, which permits use, sharing, adaptation, distribution and reproduction in any medium or format, as long as you give appropriate credit to the original author(s) and the source, provide a link to the Creative Commons license, and indicate if changes were made. The images or other third party material in this article are included in the article's Creative Commons license, unless indicated otherwise in a credit line to the material. If material is not included in the article's Creative Commons license and your intended use is not permitted by statutory regulation or exceeds the permitted use, you will need to obtain permission directly from the copyright holder. To view a copy of this license, visit http://creativecommons.org/ licenses/by/4.0/.

(C) The Author(s) 2020 